\title{
Seminal miRNA Relationship with Apoptotic Markers and Oxidative Stress in Infertile Men with Varicocele
}

\author{
Taymour Mostafa, ${ }^{1}$ Laila A. Rashed, ${ }^{2}$ Nashaat I. Nabil, ${ }^{3}$ Ihab Osman, ${ }^{1}$ \\ Rashad Mostafa, ${ }^{4}$ and Mohamed Farag ${ }^{3}$ \\ ${ }^{1}$ Department of Andrology \& Sexology, Faculty of Medicine, Cairo University, Cairo, Egypt \\ ${ }^{2}$ Department of Medical Biochemistry, Faculty of Medicine, Cairo University, Cairo, Egypt \\ ${ }^{3}$ Department of Andrology and Sexology, Faculty of Medicine, Beni Suef University, Beni Suef, Egypt \\ ${ }^{4}$ Department of Andrology and Sexology, Faculty of Medicine, Suez Canal University, Ismailia, Egypt
}

Correspondence should be addressed to Taymour Mostafa; taymour1155@link.net

Received 9 August 2016; Accepted 16 November 2016

Academic Editor: Robert Stoehr

Copyright (C) 2016 Taymour Mostafa et al. This is an open access article distributed under the Creative Commons Attribution License, which permits unrestricted use, distribution, and reproduction in any medium, provided the original work is properly cited.

\begin{abstract}
Aim. This study aimed to assess seminal miRNA relationship with seminal apoptotic markers and oxidative stress (OS) in infertile men associated with varicocele $(\mathrm{Vx})$. Methods. In all, 220 subjects were divided into the following groups: fertile normozoospermic men, fertile normozoospermic men with Vx, infertile oligoasthenoteratozoospermic (OAT) men without Vx, and infertile OAT men with Vx. They were subjected to history taking, clinical examination, and semen analysis. In their semen, the following were estimated: miRNA-122, miRNA-181a, and miRNA-34c5 using quantitative real-time PCR, apoptotic markers (BAX, BCL2) protein expression, and OS markers [malondialdehyde (MDA) and glutathione peroxidase (GPx)]. Results. The mean levels of seminal miRNA-122, miRNA-181a, and miRNA-34c5 were significantly reduced in infertile OAT men with Vx compared with other groups coupled with Vx grade and Vx bilaterality. Seminal miRNA-122, miRNA-181a, and miRNA-34c5 were positively correlated with sperm concentration, total sperm motility, sperm normal morphology, seminal GPx, and seminal BCL2 and negatively correlated with seminal MDA and seminal BAX. Conclusions. Seminal miRNA-122, miRNA-181a, and miRNA-34c5 are decreased in infertile OAT men with Vx associated with increased Vx grade and Vx bilaterality. In addition, they are positively correlated with sperm parameters and negatively correlated with OS, apoptotic markers.
\end{abstract}

\section{Introduction}

MicroRNAs (miRNA) are a family of small noncoding RNAs of 22 nucleotides that regulate posttranscriptional gene silencing through base pair binding to untranslated region of their target mRNAs [1]. Several miRNAs are implicated in regulating $\mathrm{B}$-cell differentiation and $\mathrm{T}$-cell receptor signaling, whereas others are associated with inflammation, innate immune responses, and apoptosis [2].

miRNAs were initially detected in the human spermatozoa by Ostermeier et al. [3]. They may participate in the spermatogenesis process because many miRNAs are produced plentifully in male germ cells. Wang et al. [4] speculated that seminal miRNAs, as minimal invasive biomarkers, could provide useful information about gene expression in the male reproductive system. Therefore, seminal miRNAs possessed immense potential for forensic body fluid identification being expressed in a tissue specific manner and being less prone to degradation [5].

Varicocele $(\mathrm{Vx})$ is defined as dilated veins of the pampiniform plexus in the spermatic cord. Several studies tried to explicate how Vx impairs male fertility including scrotal hyperthermia, retrograde flow of metabolites, Leydig cell dysfunction, hypoxia, impaired testicular artery perfusion, and disrupted blood-testis barrier [6, 7]. Additionally, Vx associated cases were verified to have increased seminal OS as well as seminal apoptotic markers [8-10].

This study aimed to assess seminal miRNA relationship with seminal apoptotic markers and OS in infertile men with $\mathrm{Vx}$. 


\section{Materials and Methods}

This case-controlled study included 220 men that were consecutively recruited from the University Hospital, after institutional review board approval and informed consent. Exclusion criteria were radiation, smoking, leukocytospermia, gonadotoxin, abnormal karyotyping, and immunological factors. These subjects were divided into fertile normozoospermic volunteers $(n=52)$, fertile normozoospermic men with $\mathrm{Vx}(n=43)$, infertile OAT men without $\mathrm{Vx}$ $(n=62)$, and infertile OAT men with $\mathrm{Vx}(n=63)$. Fertile groups included men who initiated at least one natural pregnancy in the previous year whereas infertile groups included men from couples that failed to conceive after at least 1 year of regular unprotected intercourse in spite of normal female factor. Normozoospermia means sperm count $>15$ million sperm $/ \mathrm{ml}$, sperm motility $>40 \%$, and normal sperm morphology $>4 \%$ whereas OAT means sperm count $<15$ million sperm $/ \mathrm{ml}$, sperm motility $<40 \%$, and normal sperm morphology $<4 \%$. All participants were subjected to history taking, clinical examination, and semen analysis. Vx diagnosis was performed with the subject in the standing position in a temperature-controlled room by inspection then palpation with/without Valsalva maneuver aided with high resolution Doppler ultrasonography.

Semen samples were collected twice, 2 weeks apart after 4-5 days of sexual abstinence. These samples were examined after liquefaction according to the WHO guidelines [11]. In the semen, the following were estimated: miRNA-122, miRNA-181a, and miRNA-34c5 by quantitative real-time PCR, apoptotic markers (BAX, BCL2) protein expression, and OS markers (malondialdehyde (MDA), glutathione peroxidase (GPx).

2.1. miRNA Assay. Semen samples were centrifuged for $5 \mathrm{~min}$ at $13,400 \mathrm{~g}$ and then $100 \mathrm{ml}$ of the supernatant seminal plasma was used for total RNA isolation using equal volume of TRIzol Reagent (Life Technologies Co., Carlsbad, CA, USA). Three steps of phenol/chloroform purification were added in order to get rid of proteins. The concentration and purity of RNA were determined using NanoDrop ${ }^{\circledR}$ ND-1000 whereas its quality was verified by denaturing agarose gel electrophoresis. Reverse transcription was performed on $5 \mathrm{ng}$ of total RNA in a final volume of $20 \mu \mathrm{l}$ (incubated for $60 \mathrm{~min}$ at $37^{\circ} \mathrm{C}$ and $5 \mathrm{~min}$ at $95^{\circ} \mathrm{C}$ and maintained at $4^{\circ} \mathrm{C}$ ) using the miRNeasy Reverse Transcription kit (Qiagen, Valencia, CA, USA).

miR-122, miR-181a, and miR-34c-5 were evaluated by qRT-PCR analysis. cDNA template was mixed with SYBER green master mix (Qiagen, Valencia, CA, USA) in a final volume of $25 \mu \mathrm{l}$. The mix was added to a custom 96-well miScript miRNA PCR array plate enriched with forward and reverse miRNA-specific Primers. Real-time PCR reactions were performed using Step one plus system (Applied Biosystems, Foster city, CA, USA) with the following conditions: $95^{\circ} \mathrm{C}$ for $15 \mathrm{~min}$, then 40 cycles at $94^{\circ} \mathrm{C}$ for $15 \mathrm{sec}, 55^{\circ} \mathrm{C}$ for $30 \mathrm{sec}$, and $70^{\circ} \mathrm{C}$ for $34 \mathrm{sec}$. Expression of miRNAs was reported as the $\Delta \mathrm{Ct}$ value calculated by subtracting the CT values of miRNA SNORD68 from the CT values of the target
miRNAs. The $2^{-\Delta \Delta(C t)}$ method was used to determine the relative quantitative levels of individual miRNAs [12].

2.1.1. Estimation of Seminal MDA [13-15]. Thiobarbituric acid method was used, whereas $100 \mu \mathrm{l}$ of seminal plasma was added to $0.9 \mathrm{ml}$ distilled water into a glass tube. Then $0.5 \mathrm{ml}$ of thiobarbituric acid reagent $(0.67 \mathrm{~g}$ 2-thiobarbituric acid dissolved in $100 \mathrm{ml}$ distilled water with $0.5 \mathrm{~g} \mathrm{NaOH}$ and $100 \mathrm{ml}$ glacial acetic acid) was added and heated for 1 hour in a water bath. After cooling, the tubes were centrifuged for $10 \mathrm{~min}$ at $4000 \mathrm{~g}$ and the supernatant absorbance was read on a spectrophotometer at $534 \mathrm{~nm}$.

2.1.2. Estimation of Seminal GPx [16]. Ransel glutathione peroxidase kit (Randox Labs, Crumlin, UK) was used where GPx catalyzes the oxidation of glutathione with cumene hydroperoxide. In presence of glutathione reductase and nicotinamide adenine dinucleotide phosphate (reduced form) (NADPH), glutathione disulphide is converted into glutathione with a concomitant oxidation of NADPH to $\mathrm{NADP}^{+}$. The decrease in the absorbance was measured at $340 \mathrm{~nm}$ at $37^{\circ} \mathrm{C}(\mathrm{pH} 7.2)$.

2.1.3. Seminal BAX and BCL2 Protein Expression [17]. Three hundred $\mu \mathrm{L}$ seminal plasma was vortexed with $3 \mu \mathrm{L}$ proteaseinhibitor cocktail (Sigma-Aldrich, Saint Louis, MO, USA) and centrifuged at $2000 \mathrm{~g}$ for $10 \mathrm{~min}$; then the supernatant was centrifuged for $20 \mathrm{~min}$ at $10000 \mathrm{~g}$ at $4^{\circ} \mathrm{C}$. For protein expression Western blot assay, seminal plasma was diluted at $1: 1$ with prechilled phosphate-buffered solution containing $1.0 \%$ $(\mathrm{v} / \mathrm{v})$ protease-inhibitor cocktail. The concentration of diluted seminal proteins was measured using the bicinchoninic acid method using bovine serum albumin as a standard. Fifty $\mu \mathrm{g}$ of protein was loaded on $12 \%$ sodium dodecyl sulfate polyacrylamide gel electrophoresis and was electrotransferred to nitrocellulose membranes using a Bio-Rad semidry transfer system. All gels were equilibrated in transfer buffer $\left(0.125 \mathrm{M}\right.$ Tris, $0.096 \mathrm{M}$ glycine) for 1 hour at $4^{\circ} \mathrm{C}$ and then transferred at $15 \mathrm{~V}$ for $20 \mathrm{~min}$. Immuno-detection of the Western blot was conducted by incubating the membranes with a blocking buffer containing $5 \%$ nonfat milk in Trisbuffered saline tween-20 (TBST) buffer $(0.5 \mathrm{mmol} / \mathrm{L}$ Tris$\mathrm{HCl}, 45 \mathrm{mmol} / \mathrm{L} \mathrm{NaCl}$, and $0.05 \%$ Tween 20 (pH 7.4)). The membranes were incubated at $4^{\circ} \mathrm{C}$ overnight with $\mathrm{BAX}$ and BCL2 primary antibodies (Stressgen, San Diego, CA, USA) at 1:3000 and 1:5000 dilutions then washed with TBST buffer and incubated with horseradish peroxidase-labeled rabbit anti-mouse IgG antibody (Santa Cruz Biotechnology, Santa Cruz, CA, USA) for 2 hours. The membranes were washed twice for $20 \mathrm{~min}$ with TBST and once with TBS. Antibody binding to proteins was visualized with enhanced chemiluminescence detection system (Amersham, Arlington Heights, IL, USA). Densitometric analysis of BAX and BCL2 bands was carried out using the Gel Image Scan System (Tianren, Shanghai, China) expressed relative to beta actin.

2.2. Statistical Analysis. Statistical analysis was carried out using the Windows software SPSS version 21 (SPSS Inc., Chicago, IL, USA). One way ANOVA test was used for 
TABLE 1: Comparison of different parameters in the investigated groups (mean $\pm \mathrm{SD}$, range).

\begin{tabular}{lcccc}
\hline & Fertile men & Fertile men with Vx & OAT men & OAT men with Vx \\
\hline$n$ & 52 & 43 & 62 & 63 \\
Age (years) & $30.0 \pm 2.9(25-37)$ & $30.3 \pm 3.8(25-39)$ & $29.3 \pm 3.3(23-36)$ & $29.0 \pm 3.6(24-39)$ \\
Sperm count $\left(10^{6} / \mathrm{ml}\right)$ & $70.2 \pm 0.6(21-97)$ & $68.1 \pm 7.5(31-86)$ & $7.9 \pm 3.1(1.6-12)^{\mathrm{a}, \mathrm{b}}$ & $7.4 \pm 2.8(2-12)^{\mathrm{a}, \mathrm{b}}$ \\
Sperm motility (\%) & $60.4 \pm 6.7(45-71)$ & $58.8 \pm 5.2(48-65)$ & $21.5 \pm 7.9(9-36)^{\mathrm{a}, \mathrm{b}}$ & $14.6 \pm 6.6(5-25)^{\mathrm{a}, \mathrm{b}}$ \\
Sperm normal morphology (\%) & $6.8 \pm 1.0(6-9)$ & $5.93 \pm 0.82(4-7)$ & $2.7 \pm 1(0-4)^{\mathrm{a}, \mathrm{b}}$ & $2.8 \pm 0.5(1-3)^{\mathrm{a}, \mathrm{b}}$ \\
Seminal BAX & $1.24 \pm 0.4(0.1-2.04)$ & $7.0 \pm 1.5(4-9.6)^{\mathrm{a}}$ & $9.7 \pm 1(7.7-12)^{\mathrm{a}, \mathrm{b}}$ & $13.6 \pm 2.6(10.1-17.6)^{\mathrm{a}, \mathrm{b}, \mathrm{c}}$ \\
Seminal BCl2 & $1.3 \pm 0.4(0.44-1.9)$ & $0.82 \pm 0.5(0.23-1.78)^{\mathrm{a}}$ & $0.45 \pm 0.1(0.11-0.62)^{\mathrm{a}, \mathrm{b}}$ & $0.23 \pm 0.1(0.12-0.46)^{\mathrm{a}, \mathrm{b}, \mathrm{c}}$ \\
Seminal GPx (U/ml) & $57.5 \pm 4.5(39-68.7)$ & $33.5 \pm 2.5(28.9-39.1)^{\mathrm{a}}$ & $27.9 \pm 3.8(15.4-37.4)^{\mathrm{a}, \mathrm{b}}$ & $19.9 \pm 3.9(14.2-28.2)^{\mathrm{a}, \mathrm{b}, \mathrm{c}}$ \\
Seminal MDA (nmol/ml) & $1.2 \pm 0.4(0.09-2.03)$ & $6.2 \pm 1.6(2.7-9.12)^{\mathrm{a}}$ & $7.6 \pm 1.6(5-11)^{\mathrm{a}, \mathrm{b}}$ & $10.6 \pm 1.9(6.8-14.2)^{\mathrm{a}, \mathrm{b}, \mathrm{c}}$ \\
Seminal miRNA-122 & $54.3 \pm 4.2(40.7-68.4)$ & $33.6 \pm 4.5(25.8-43.7)^{\mathrm{a}}$ & $26.8 \pm 5.1(16.9-42)^{\mathrm{a}, \mathrm{b}}$ & $20.0 \pm 4.7(10.2-33.8)^{\mathrm{a}, \mathrm{b}, \mathrm{c}}$ \\
Seminal miRNA-181-a & $23.1 \pm 3.0(16.4-30.6)$ & $14.9 \pm 2.5(9.8-20.3)^{\mathrm{a}}$ & $13.5 \pm 3.2(7.1-25.8)^{\mathrm{a}, \mathrm{b}}$ & $7.4 \pm 1.9(4.03-10.8)^{\mathrm{a}, \mathrm{b}, \mathrm{c}}$ \\
Seminal miRNA -34c-5 & $10.9 \pm 1.8(7.1-14.8)$ & $8.3 \pm 1.6(5.04-11.6)^{\mathrm{a}}$ & $6.7 \pm 2.0(3.2-10.8)^{\mathrm{a}, \mathrm{b}}$ & $2.8 \pm 1(1.7-4.8)^{\mathrm{a}, \mathrm{b}, \mathrm{c}}$ \\
\hline
\end{tabular}

${ }^{\mathrm{a}}$ Statistical difference compared with fertile men (controls).

${ }^{\mathrm{b}}$ Statistical difference compared with fertile men with Vx.

${ }^{\mathrm{c}}$ Statistical difference compared with OAT without $\mathrm{Vx}$.

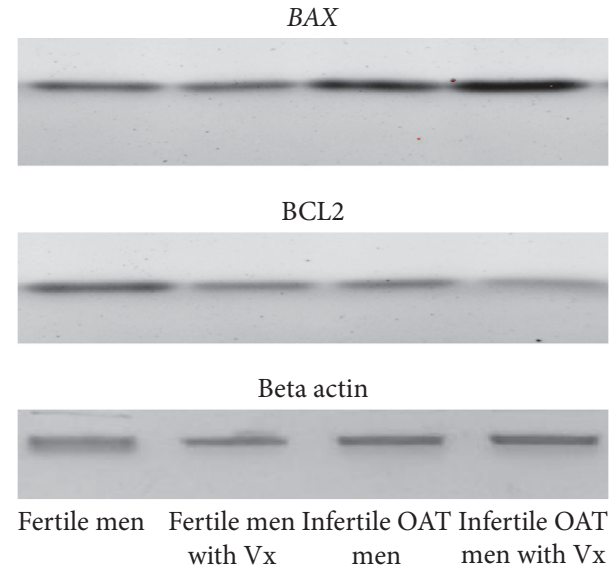

FIGURE 1: Western blot image of seminal BAX and BCL2 expressed relative to beta actin in the investigated groups.

comparisons with Tukey post hoc test whereas Pearson correlation was applied for relations between tested variables. $P$ value $<0.05$ was set as statistically significant.

\section{Results}

Seminal miRNA-122, miRNA-181a, and miRNA-34c5, seminal GPx enzyme, and seminal BCL2 were significantly reduced whereas seminal MDA and seminal BAX were significantly increased in infertile OAT men with Vx compared with other groups (Table 1, Figure 1). Seminal miRNA-122, miRNA-181a, and miRNA-34c5 were significantly reduced in grade III $\mathrm{Vx}$ cases compared with other $\mathrm{Vx}$ grades and in bilateral $\mathrm{Vx}$ cases compared with unilateral $\mathrm{Vx}$ cases (Table 2).

Seminal miRNA-122, miRNA-181a, and miRNA-34c5 showed significant positive correlation with sperm concentration $(r=0.688, r=0.656, r=0.695$, and $P=0.001)$, sperm motility $(r=0.732, r=0.677, r=0.756$, and
$P=0.001)$, sperm normal morphology $(r=0.738, r=$ $0.701, r=0.719$, and $P=0.001)$, seminal GPx $(r=0.805$, $r=0.805, r=0.787$, and $P=0.001)$, and seminal BCL2 ( $r=0.740, r=0.676, r=0.727$, and $P=0.001)$ and they showed significant negative correlation with seminal MDA $(r=-0.814, r=-0.782, r=-0.781$, and $P=0.001)$ and seminal $\operatorname{BAX}(r=-0.793, r=-0.791, r=-0.780$, and $P=$ 0.001) (Figures 2-4). Seminal miRNA-122 showed significant positive correlation with seminal miRNA-181a and miRNA$34 c 5$ ( $r=0.764, r=0.728, P=0.001)$ and seminal miRNA181a showed significant positive correlation with miRNA$34 \mathrm{c5}(r=0.729, P=0.001)$ (Figure 5).

\section{Discussion}

RNA is not just a simple messenger between DNA and proteins but new roles for these molecules are lately raised, such as regulation of genome organization and gene expression. In the current study, there was a significant decrease in seminal plasma miRNA-122, miRNA-181a, and miRNA-34c5 in infertile OAT men with $\mathrm{Vx}$ compared with other investigated groups. In addition, they were positively correlated with sperm concentration, sperm motility, and sperm normal morphology and negatively correlated with seminal OS and the tested apoptotic markers.

Growing evidence has designated that miRNAs are involved in the regulation of heat and OS responses, the two raised elements contributing to Vx-related sperm dysfunction $[18,19]$. In their study, McCallie et al. [20] showed that morphologically similar blastocysts of infertile men have lowered expression of six miRNA compared with fertile men. Wang et al. [4] pointed out that seven miRNAs (miRNA34c-5p, miRNA-122, miRNA-146b-5p, miRNA-181a, miRNA374b, miRNA-509-5p, and miRNA-513a-5p) are markedly decreased in the seminal plasma of asthenozoospermic men compared with normozoospermic men. Liu et al. [21] observed high levels of miRNA-34c in adult pachytene spermatocytes and round spermatids being important to the 


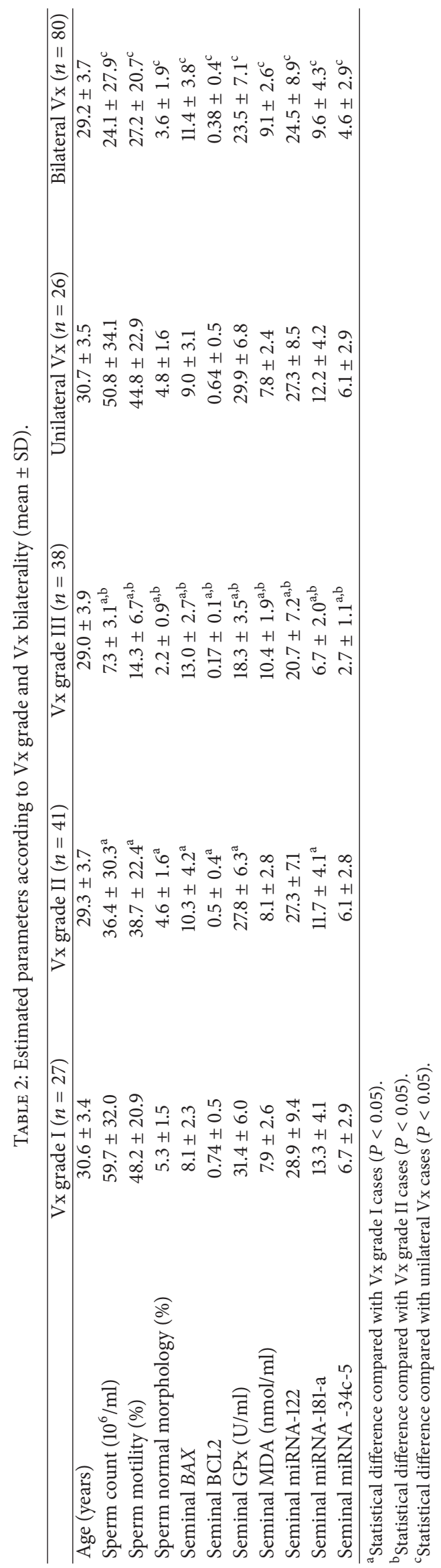




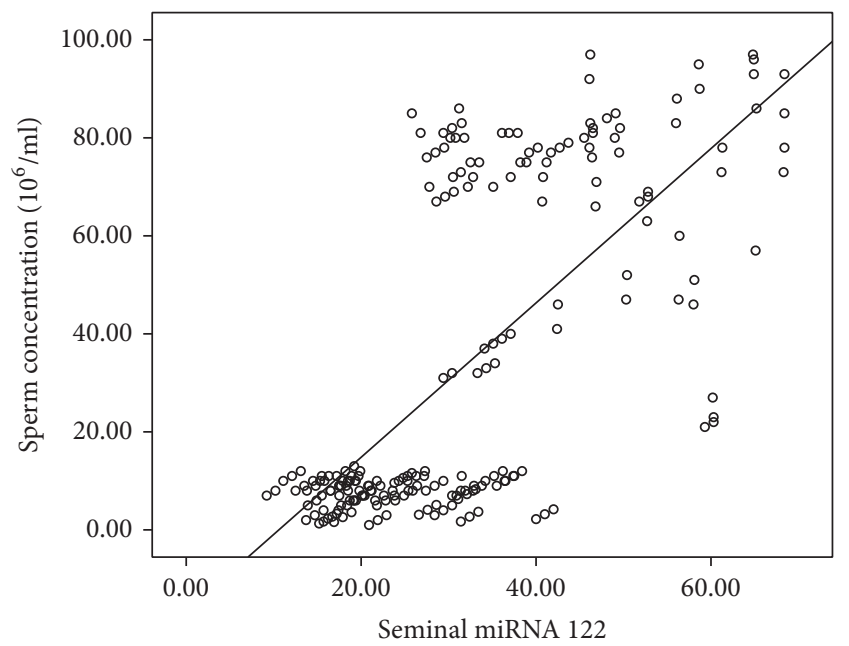

(a)

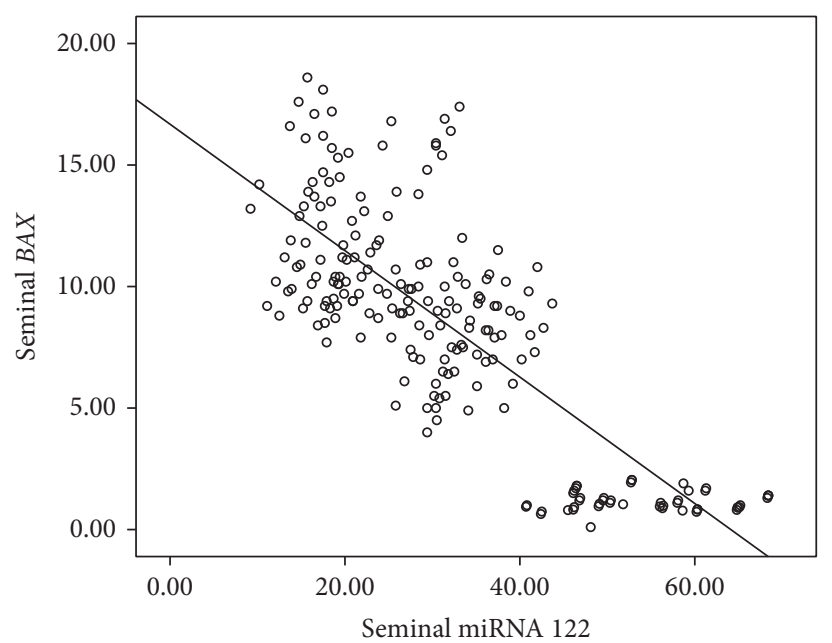

(c)

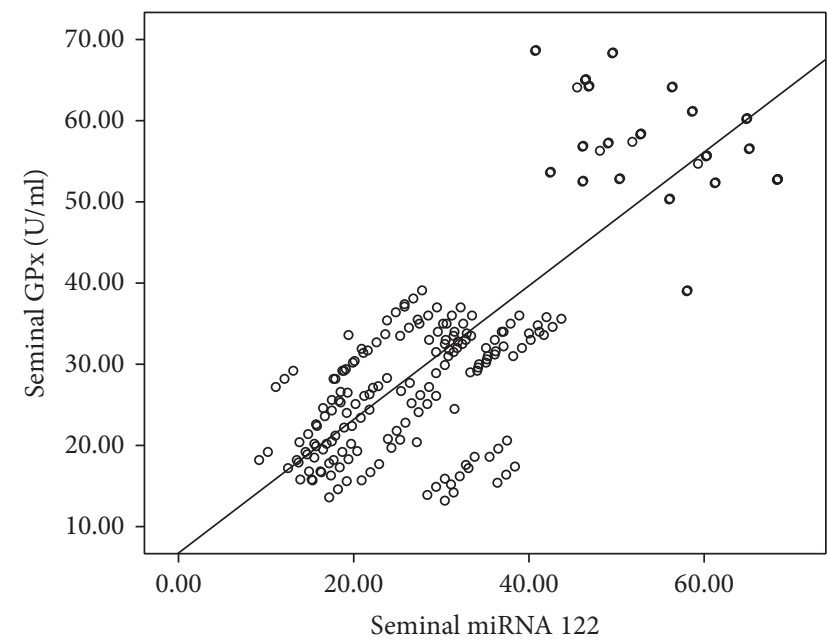

(e)

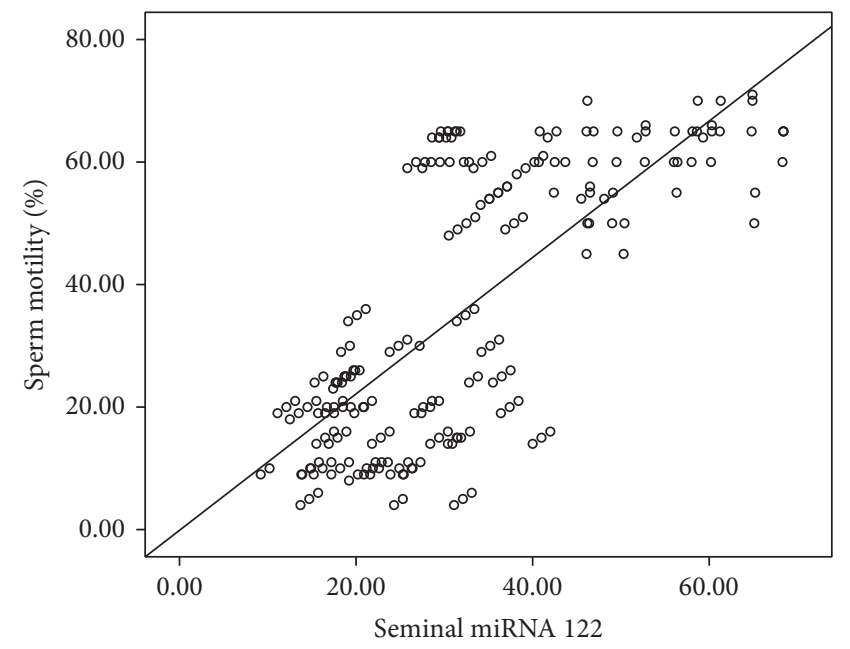

(b)

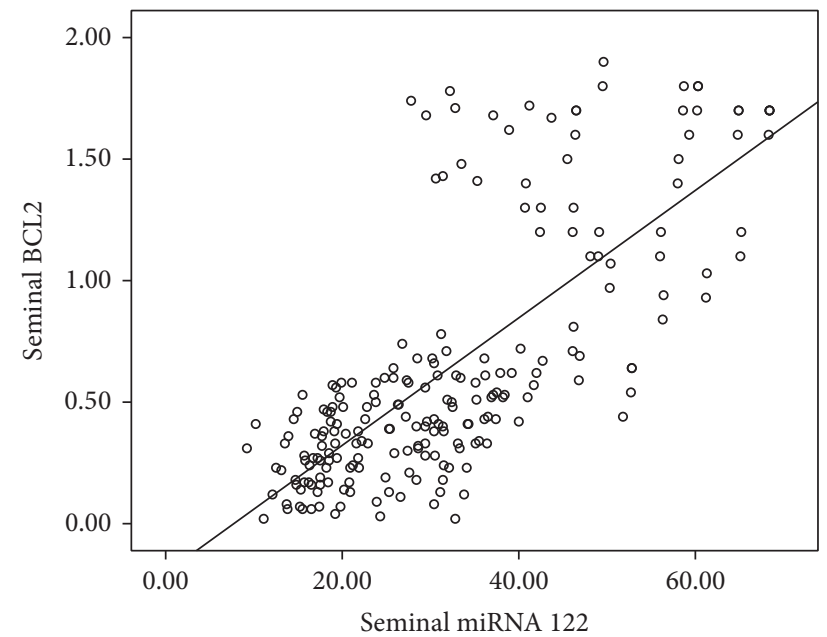

(d)

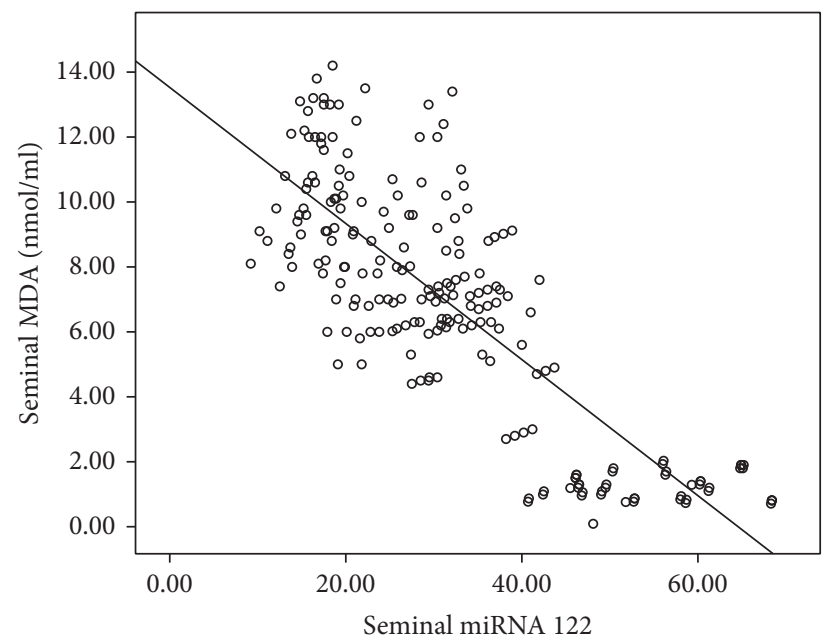

(f)

FIgURE 2: Seminal miRNA-122 (X-axis) significant positive correlations with sperm concentration (a), (b) sperm motility, (e) seminal GPx, and (d) seminal BCL2 and significant negative correlations with (c) seminal BAX and (f) seminal MDA. 


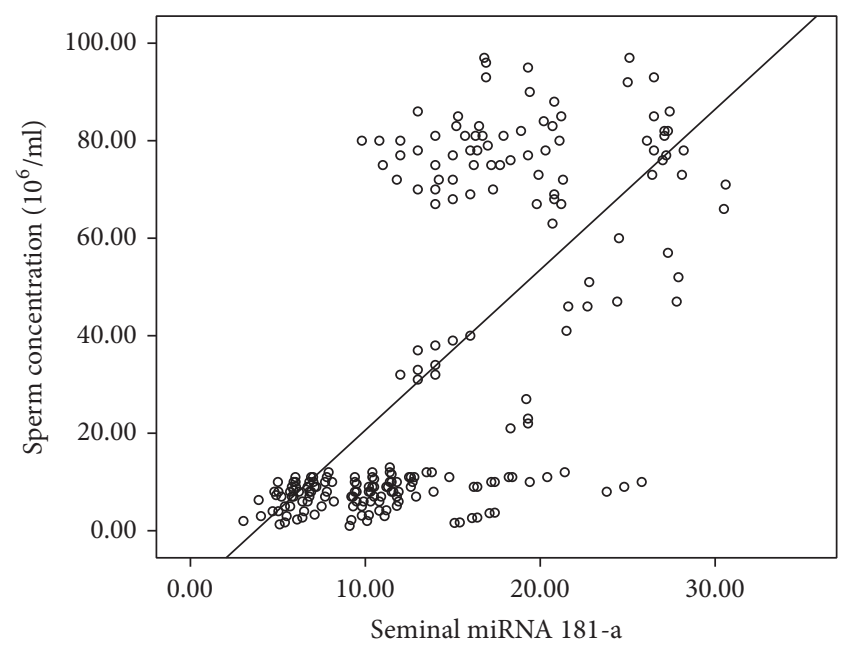

(a)

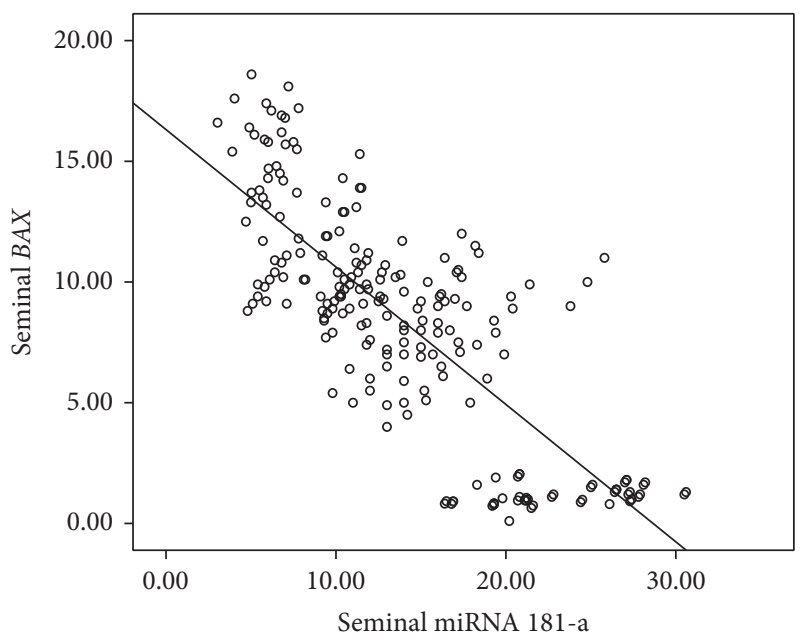

(c)

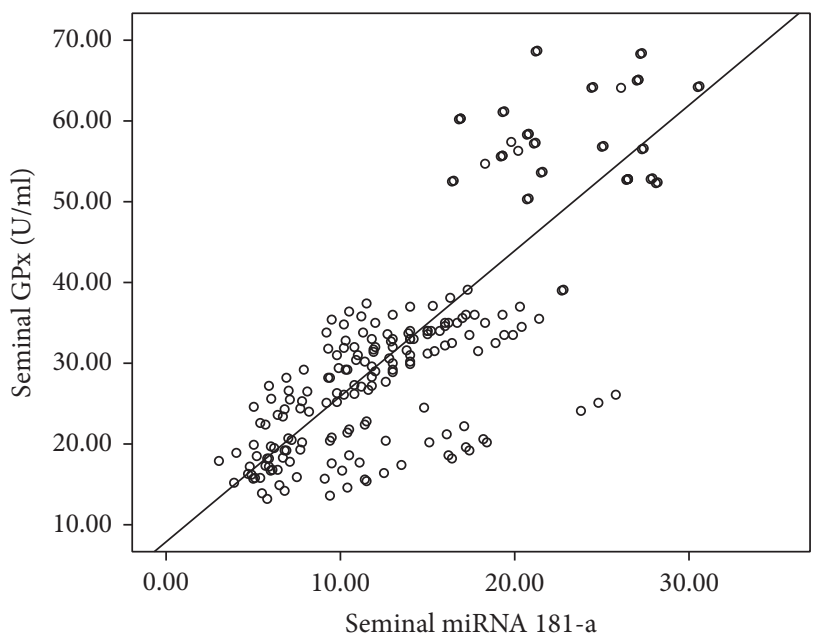

(e)

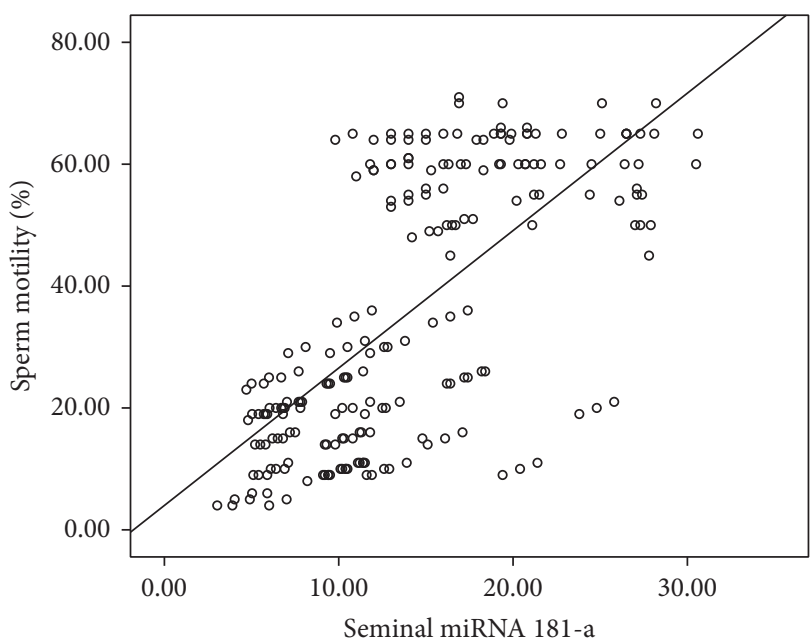

(b)

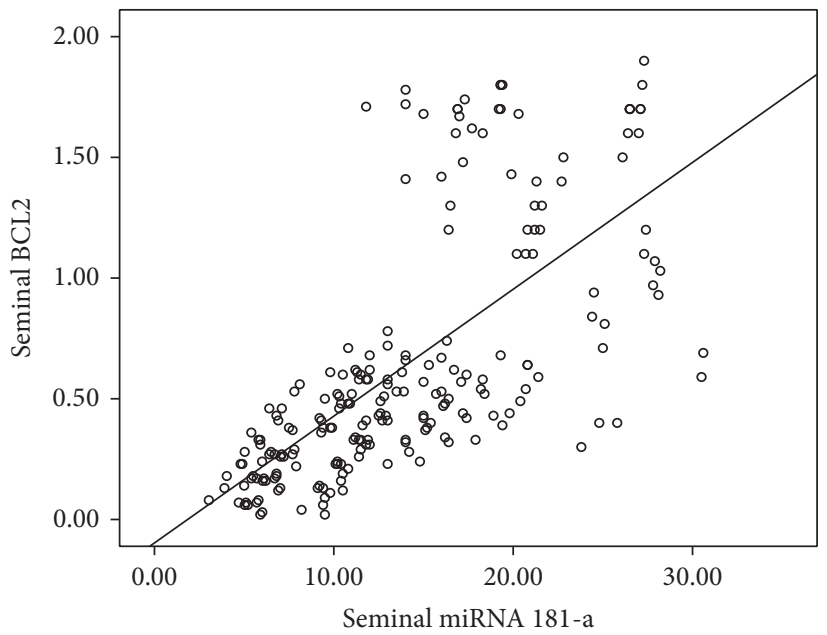

(d)

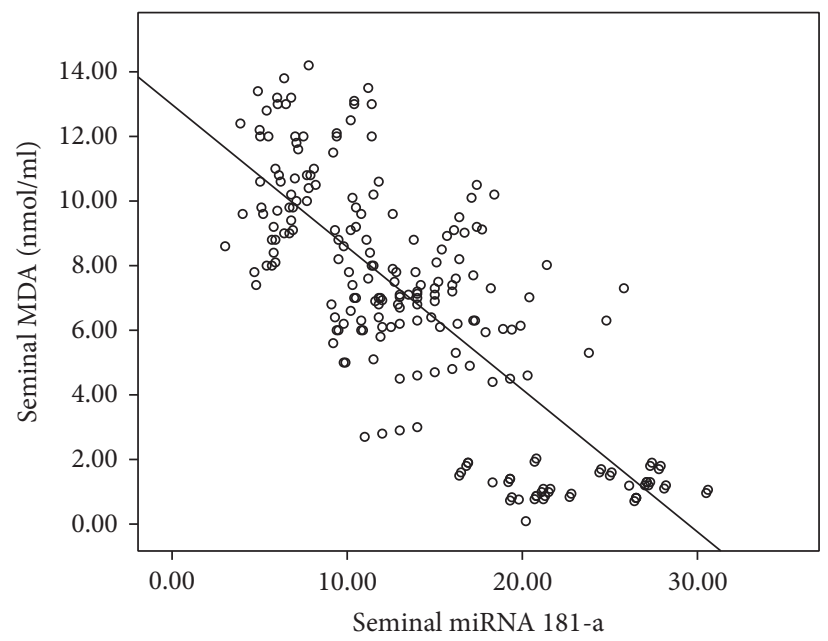

(f)

Figure 3: Seminal miRNA-181a ( $X$-axis) significant positive correlations with (a) sperm concentration, (b) sperm motility, (e) seminal GPx, and (d) seminal BCL2 and significant negative correlations with (c) seminal BAX and (f) seminal MDA. 


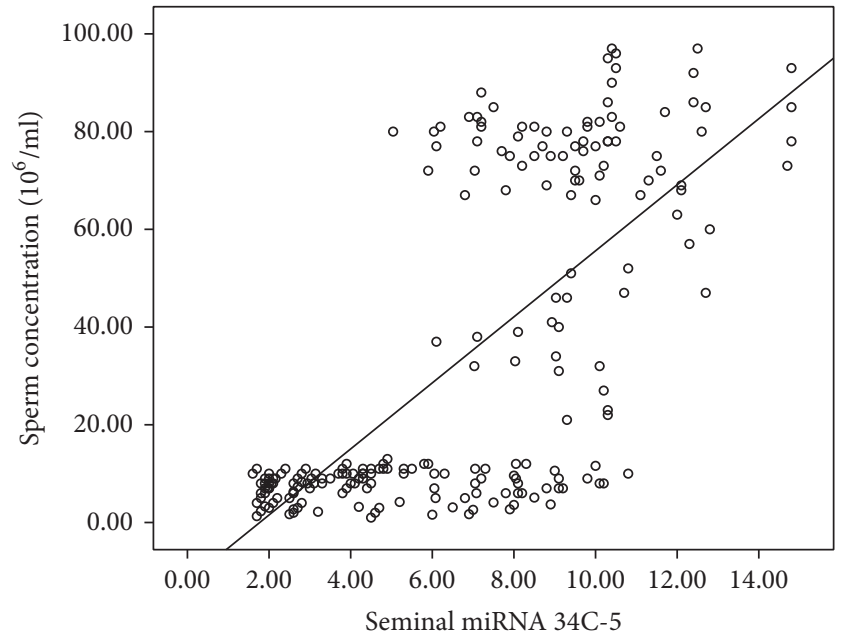

(a)

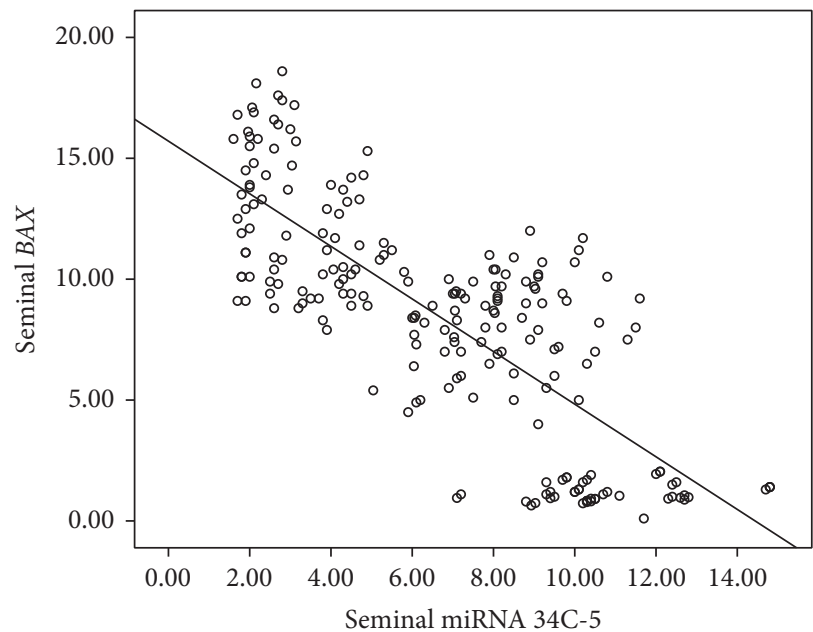

(c)

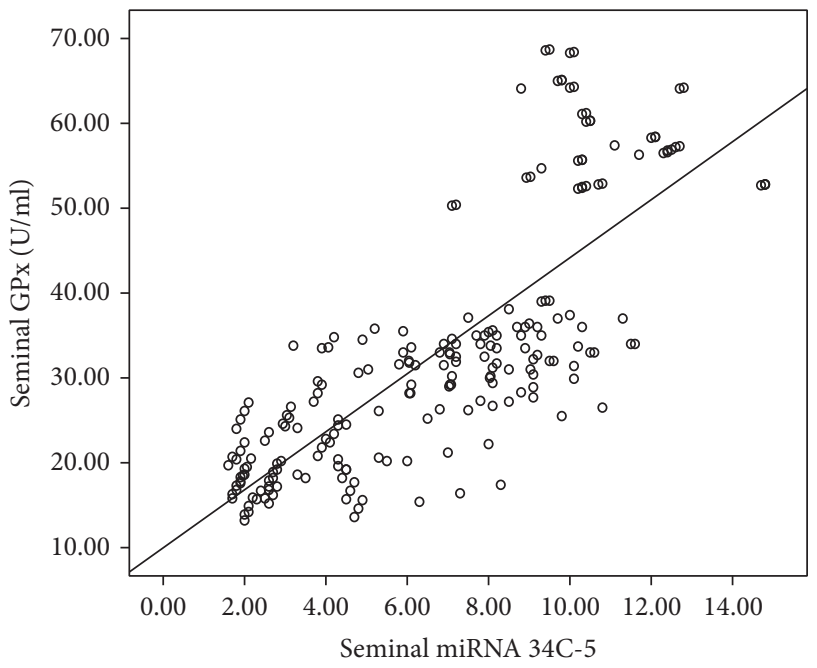

(e)

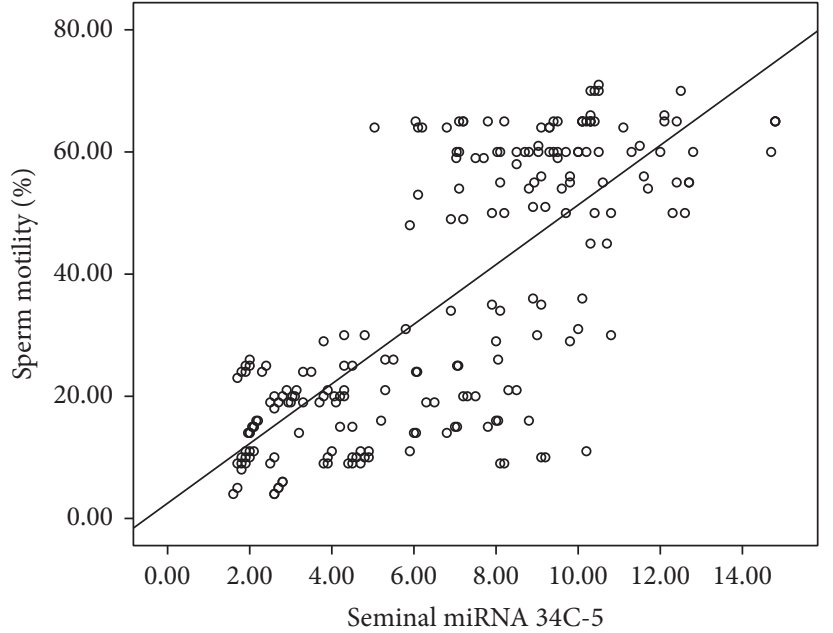

(b)

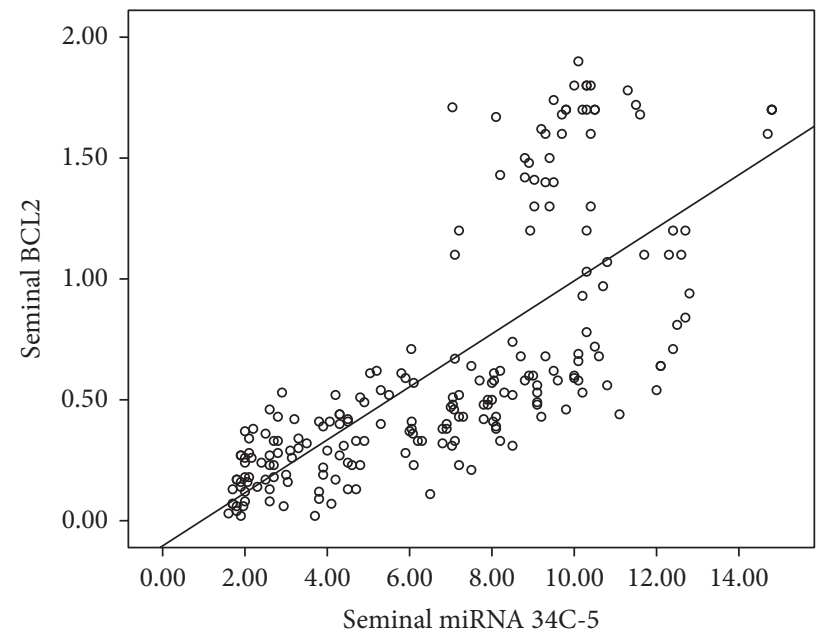

(d)

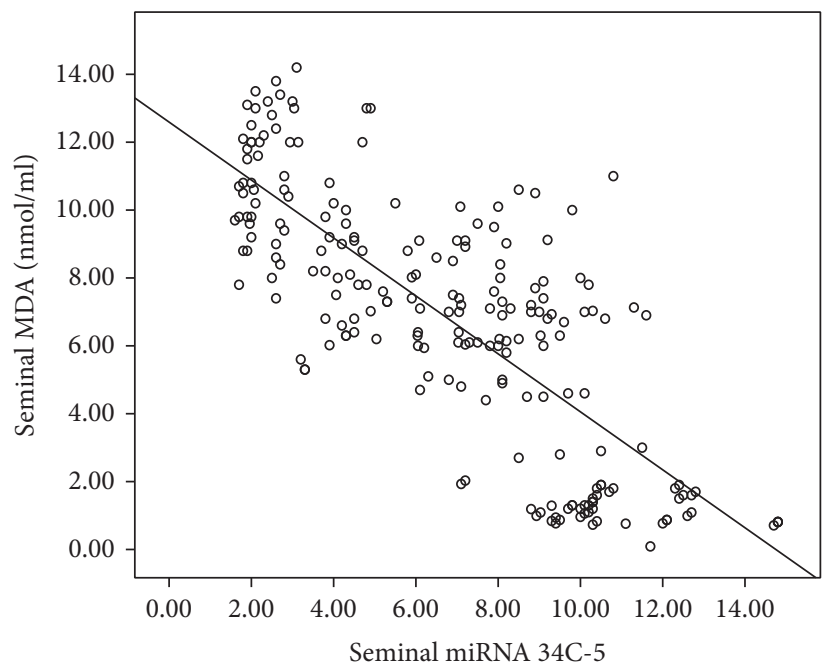

(f)

FIGURE 4: Seminal miRNA-34c5 ( $X$-axis) significant positive correlations with (a) sperm concentration, (b) sperm motility, (e) seminal GPx, and (d) seminal BCL2 and significant negative correlations with seminal (c) BAX and (f) seminal MDA. 


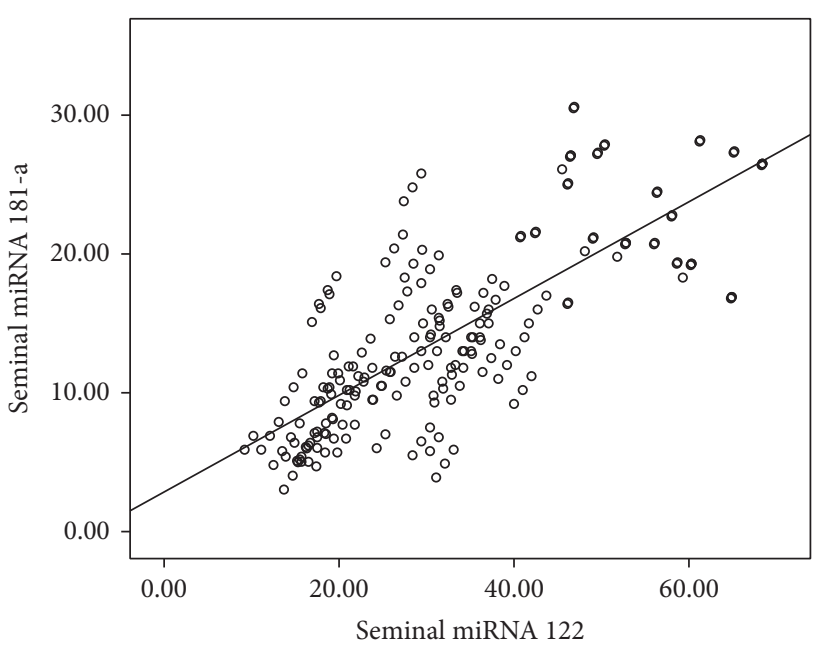

(a)

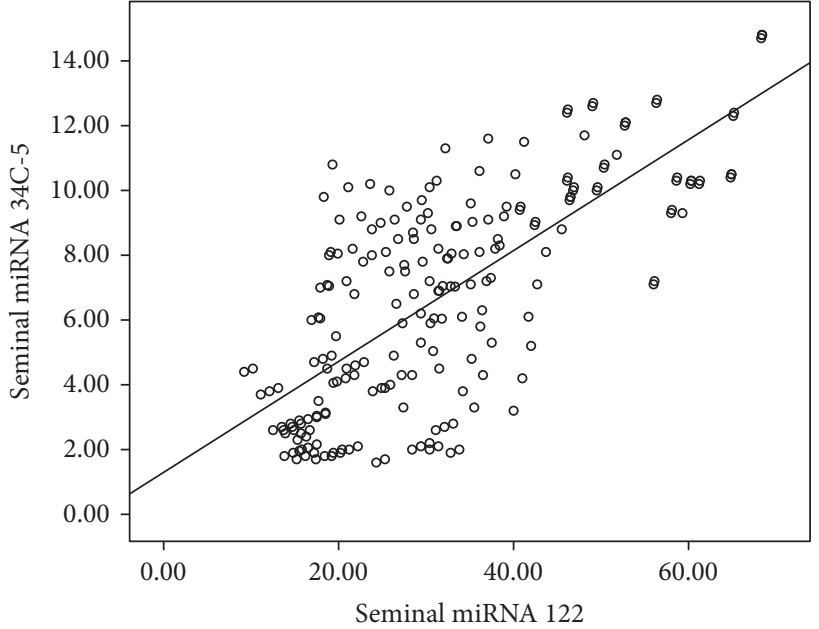

(b)

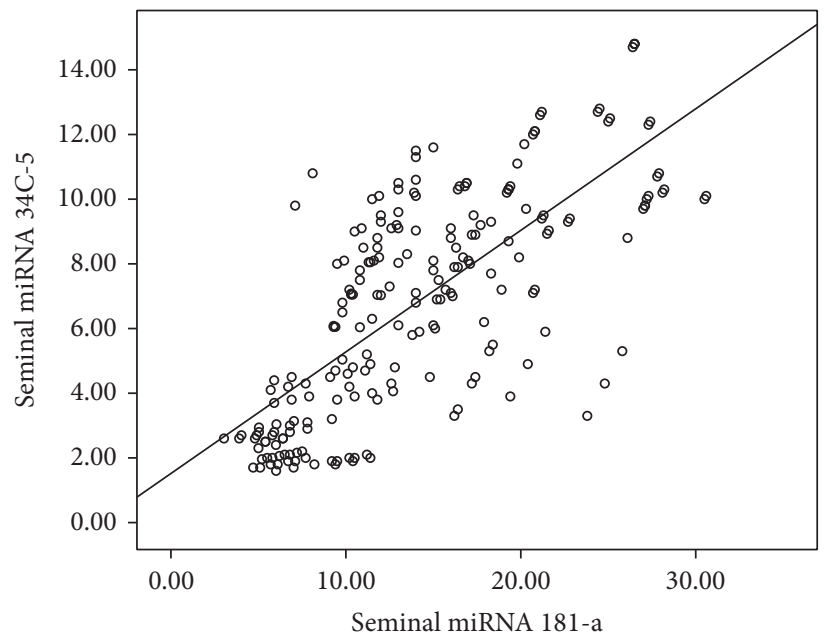

(c)

FiguRE 5: Seminal miRNA-122 significant positive correlation with seminal miRNA-181a (a) and miRNA-34c5 (b). Significant positive correlations of seminal miRNA-181a and miRNA-34c5 (c).

first cell division via modulating BCL2 expression, which is a direct target of miRNA-34c. Ji et al. [22] pointed out that the expression of miRNA-15a was significantly decreased in the spermatozoa of infertile men associated with $\mathrm{Vx}$ compared with fertile controls repressing expressed stressinduced chaperone protein. In this context, Wenhao et al. [23] showed that miRNAs are involved in regulating the expression of HSP40, HSP70, and HSP90 affecting sperm function with significant differences in expression between oligoasthenozoospermic and normozoospermic men.

The estimated seminal miRNAs showed significant decrease with increased Vx grade and Vx bilateral cases. Different studies pointed out the deleterious effects with increased Vx grade and its bilaterality. Vivas-Acevedo et al. [24] and Mostafa et al. [25] showed that elevated Vx grade could determine the extent of altered semen quality. Cocuzza et al. [26] pointed out that as Vx grade increased, seminal OS increases and sperm concentration decreases. Also, Mostafa et al. [27] observed that seminal OS is related to increased Vx grade as well as bilaterality in infertile OAT men associated with Vx.

The limitations of this study included the need to assess these seminal miRNAs after varicocele repair linked with semen parameters, OS, apoptotic markers, and pregnancy rates as noninvasive biomarkers for post-Vx repair seminal improvement.

It is concluded that seminal miRNA-122, miRNA-181a, and miRNA-34c5 are decreased in infertile OAT men with Vx associated with increased Vx grade and Vx bilaterality. In addition, they are positively correlated with sperm parameters and negatively correlated with OS, apoptotic markers.

\section{Competing Interests}

The authors declare no competing interests. 


\section{References}

[1] A. Abhari, N. Zarghami, V. Shahnazi et al., "Significance of microRNA targeted estrogen receptor in male fertility," Iranian Journal of Basic Medical Sciences, vol. 17, no. 2, pp. 81-86, 2014.

[2] W.-L. Jiang, Y.-F. Zhang, Q.-Q. Xia et al., "MicroRNA-19a regulates lipopolysaccharide-induced endothelial cell apoptosis through modulation of apoptosis signal-regulating kinase 1 expression," BMC Molecular Biology, vol. 16, article no. 11, 2015.

[3] G. C. Ostermeier, D. J. Dix, D. Miller, P. Khatri, and S. A. Krawetz, "Spermatozoal RNA profiles of normal fertile men," Lancet, vol. 360, no. 9335, pp. 772-777, 2002.

[4] C. Wang, C. Yang, X. Chen et al., "Altered profile of seminal plasma microRNAs in the molecular diagnosis of male infertility," Clinical Chemistry, vol. 57, no. 12, pp. 1722-1731, 2011.

[5] J.-L. Park, S.-M. Park, O.-H. Kwon et al., "Microarray screening and qRT-PCR evaluation of microRNA markers for forensic body fluid identification," Electrophoresis, vol. 35, no. 21-22, pp. 3062-3068, 2014.

[6] A. Sadek, A. S. A. Almohamdy, A. Zaki, M. Aref, S. M. Ibrahim, and T. Mostafa, "Sperm chromatin condensation in infertile men with varicocele before and after surgical repair," Fertility and Sterility, vol. 95, no. 5, pp. 1705-1708, 2011.

[7] A. Zalata, M. El-Mogy, A. Abdel-Khabir, Y. El-Bayoumy, M. El-Baz, and T. Mostafa, "Sperm caspase-9 in oligoasthenoteratozoospermic men with and without varicocele," Fertility and Sterility, vol. 96, no. 5, pp. 1097-1099, 2011.

[8] A. Hassan, E. M. El-Nashar, and T. Mostafa, "Programmed cell death in varicocele-bearing testes," Andrologia, vol. 41, no. 1, pp. 39-45, 2009.

[9] H. Zedan, A. W. M. M. El-Mekhlafi, A. M. El-Noweihi, N. E. Abd El-Azim, and T. Mostafa, "Soluble Fas and gonadal hormones in infertile men with varicocele," Fertility and Sterility, vol. 91, no. 2, pp. 420-424, 2009.

[10] G. A. Tawadrous, A. A. Aziz, and T. Mostafa, "Seminal soluble fas relationship with oxidative stress in infertile men with varicocele," Urology, vol. 82, no. 4, pp. 820-823, 2013.

[11] World Health Organization, WHO Laboratory Manual for the Examination and Processing of Human Semen, WHO Press, Geneva, Switzerland, 5th edition, 2010.

[12] T. M. Motawi, S. M. Rizk, O. G. Shaker, O. Z. Mokhtar, and D. R. Booth, "MicroRNAs as predictor markers for response to interferon treatment of chronic hepatitis C genotype- 4 in Egyptian patients," PLoS One, vol. 10, no. 3, Article ID e0121524, 2015.

[13] Z. A. Placer, L. L. Cushman, and B. C. Johnson, "Estimation of product of lipid peroxidation (malonyl dialdehyde) in biochemical systems," Analytical Biochemistry, vol. 16, no. 2, pp. 359-364, 1966.

[14] H. H. Draper, E. J. Squires, H. Mahmoodi, J. Wu, S. Agarwal, and M. Hadley, "A comparative evaluation of thiobarbituric acid methods for the determination of malondialdehyde in biological materials," Free Radical Biology and Medicine, vol. 15, no. 4, pp. 353-363, 1993.

[15] S. El-Haggar, L. Rashed, N. Y. Saleh, M. Taymour, and T. Mostafa, "Seminal osteopontin relationship with semen variables in infertile men with varicocele," Human Andrology, vol. 3, no. 4, pp. 90-93, 2013.

[16] D. E. Paglia and W. N. Valentine, "Studies on the quantitative and qualitative characterization of erythrocyte glutathione peroxidase," The Journal of Laboratory and Clinical Medicine, vol. 70, no. 1, pp. 158-169, 1967.
[17] T. Mostafa, L. Rashed, N. Nabil, and R. Amin, "Seminal BAX and BCL2 gene and protein expressions in infertile men with varicocele," Urology, vol. 84, no. 3, pp. 590-595, 2014.

[18] T. Mostafa, T. Anis, H. Imam, A. R. El-Nashar, and I. A. Osman, "Seminal reactive oxygen species-antioxidant relationship in fertile males with and without varicocele," Andrologia, vol. 41, no. 2, pp. 125-129, 2009.

[19] A. Garolla, M. Torino, P. Miola et al., "Twenty-four-hour monitoring of scrotal temperature in obese men and men with a varicocele as a mirror of spermatogenic function," Human Reproduction, vol. 30, no. 5, pp. 1006-1013, 2015.

[20] B. McCallie, W. B. Schoolcraft, and M. G. Katz-Jaffe, "Aberration of blastocyst microRNA expression is associated with human infertility," Fertility and Sterility, vol. 93, no. 7, pp. 2374-2382, 2010.

[21] W.-M. Liu, R. T. K. Pang, P. C. N. Chiu et al., "Sperm-borne microRNA-34c is required for the first cleavage division in mouse," Proceedings of the National Academy of Sciences of the United States of America, vol. 109, no. 2, pp. 490-494, 2012.

[22] Z. Ji, R. Lu, L. Mou et al., "Expressions of miR-15a and its target gene HSPA1B in the spermatozoa of patients with varicocele," Reproduction, vol. 147, no. 5, pp. 693-701, 2014.

[23] T. Wenhao, L. De-Feng, H. Kai et al., "miRNA-mediated regulation of heat shock proteins in human ejaculated spermatozoa," Turkish Journal of Medical Sciences, vol. 45, no. 6, pp. 1285-1291, 2015.

[24] G. Vivas-Acevedo, J. R. Lozano, and M. I. Camejo, "Effect of varicocele grade and age on seminal parameters," Urologia Internationalis, vol. 85, no. 2, pp. 194-199, 2010.

[25] T. Mostafa, T. Anis, A. El Nashar, H. Imam, and I. Osman, "Seminal plasma reactive oxygen species-antioxidants relationship with varicocele grade," Andrologia, vol. 44, no. 1, pp. 66-69, 2012.

[26] M. Cocuzza, K. S. Athayde, C. Alvarenga, M. Srougi, and J. Hallak, "Grade 3 varicocele in fertile men: a different entity," Journal of Urology, vol. 187, no. 4, pp. 1363-1368, 2012.

[27] T. Mostafa, L. Rashed, and M. Taymour, "Seminal cyclooxygenase relationship with oxidative stress in infertile oligoasthenoteratozoospermic men with varicocele," Andrologia, vol. 48, no. 2, pp. 137-142, 2016. 


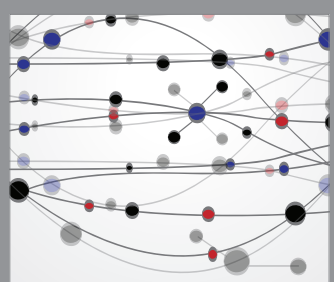

The Scientific World Journal
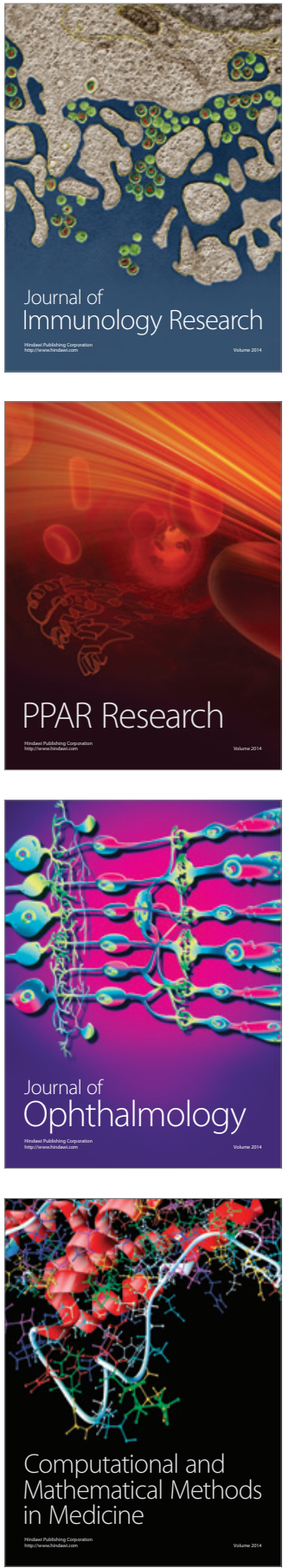

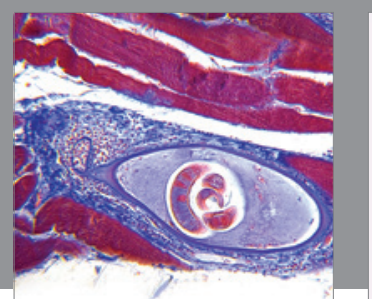

Gastroenterology Research and Practice

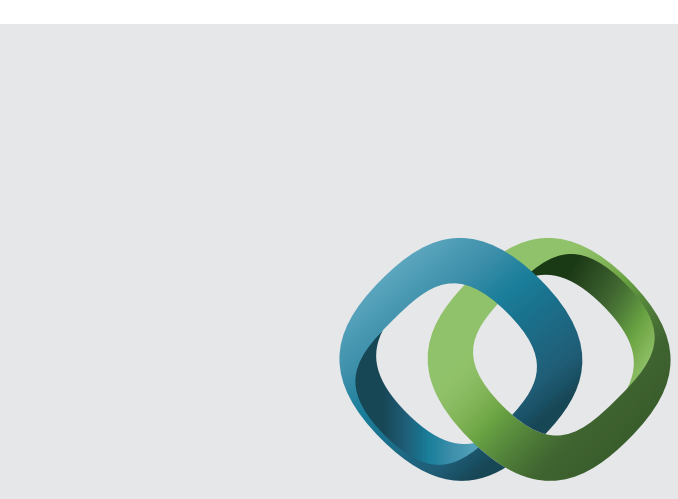

\section{Hindawi}

Submit your manuscripts at

http://www.hindawi.com
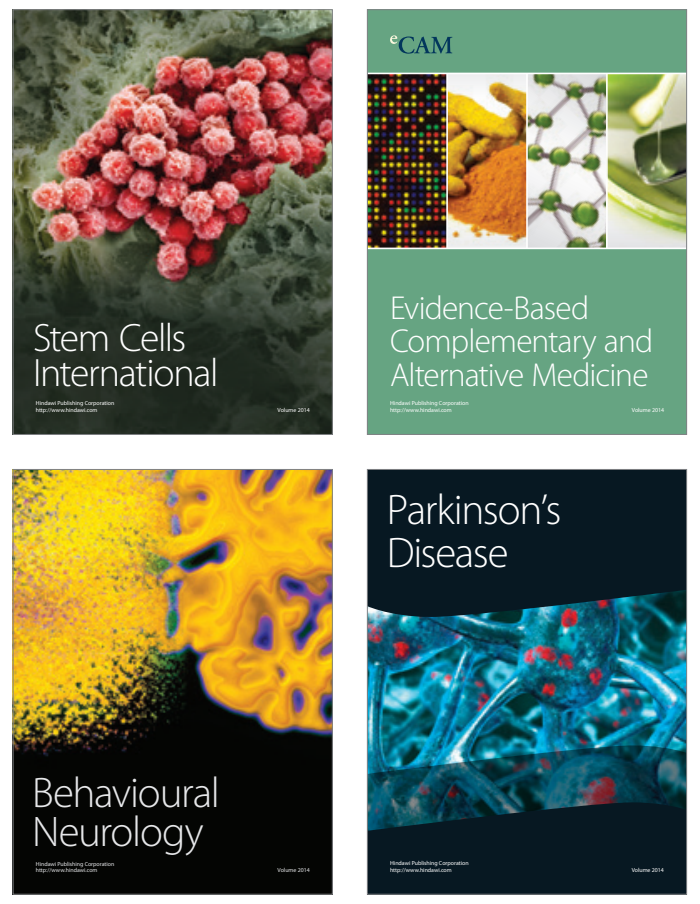
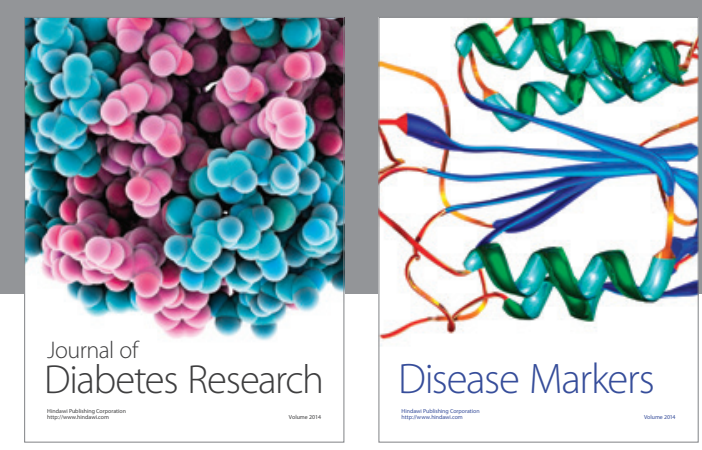

Disease Markers
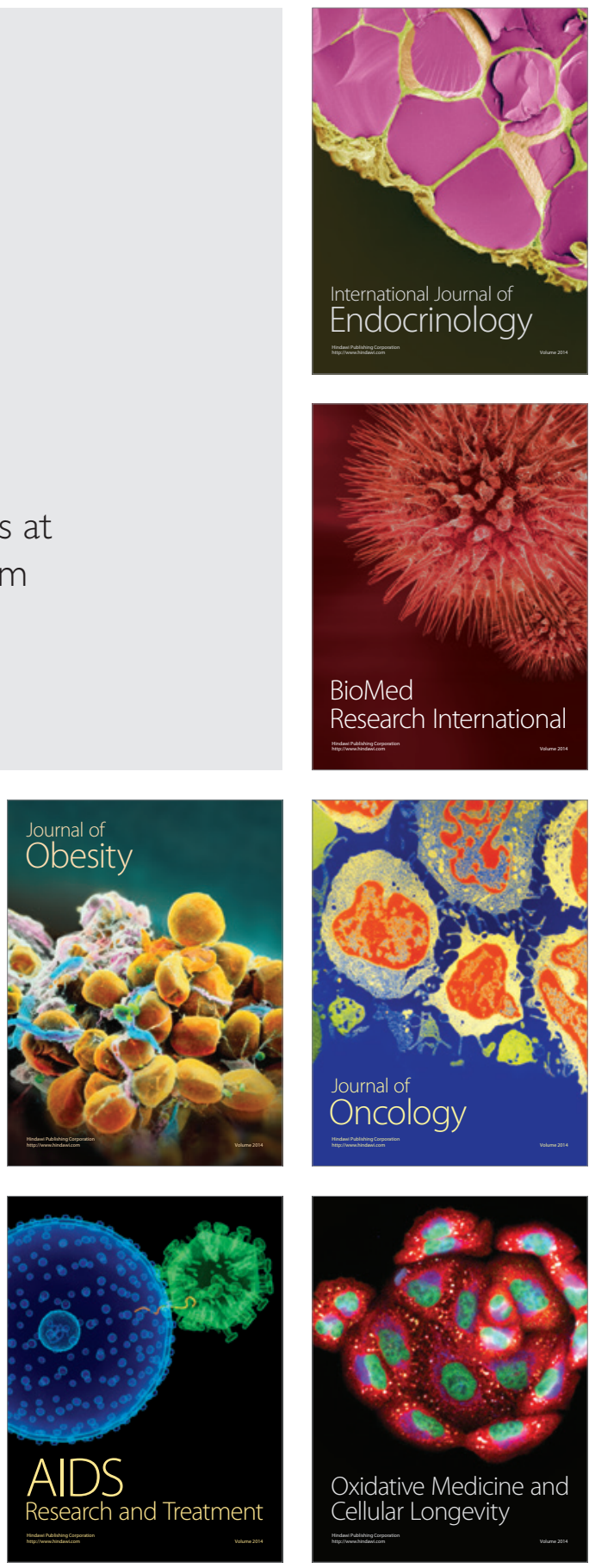